\title{
Numerical Study on Influences of Bed Resettling, Breeding Zone Orientation, and Purge Gas on Temperatures in Solid Breeders
}

\author{
Jon T. Van Lew*, Alice Ying, Mohamed Abdou \\ UCLA, MAE Department, 44-114 Engineering IV, 420 Westwood Plaza, Los Angeles, CA 90095-1597, USA
}

\begin{abstract}
We apply coupled computational fluid dynamics and discrete element method (CFD-DEM) modeling tools with new numerical implementations of pebble fragmentation to study the combined effects of granular crushing and ensemble restructuring, granular fragment size, and initial packing for different breeder volume configurations. In typical solid breeder modules, heat removal from beds relies on maintaining pebblepebble and pebble-wall contact integrity. However, contact is disrupted when an ensemble responds to individually-crushed pebbles. Furthermore, restructuring of metastable packings after crushing events are, in part, dependent on gravity forces acting upon the pebbles. We investigate two representative pebble bed configurations under constant volumetric heat sources; modeling heat removed from beds via inter-particle conduction, purge gas convection, and contact between pebble beds and containers. In one configuration, heat is removed from at walls oriented parallel to the gravity vector (no gap formation possible); in the second, heat is removed at walls perpendicular to gravity, allowing for the possibility of gap formation between bed and wall. Judging beds on increase in maximum temperatures as a function of crushed pebble amount, we find that both pebble bed configurations to have advantageous features that manifest at different stages of pebble crushing. However, all configurations benefit from achieving high initial packing fractions.
\end{abstract}

Keywords: Coupled CFD-DEM, granular heat transfer, granular crushing, ceramic breeder

\section{Introduction}

Pebble beds of lithium ceramics exist in candidate designs of nuclear fusion demonstration reactors and tritium breeding modules in ITER. For optimum tritium release, ceramic pebble beds have relatively narrow operational temperature windows that must be maintained. Bed temperatures are regulated via coolant running through the structural material, heat is transported from bed to structure, in part, from pebble/wall contact conduction. Therefore maintaining physical contact between granular material and structural material is necessary for heat outflow from the bed. Temperature prediction and control is of utmost importance for reliable tritium release and energy production in a fusion reactor's ceramic pebble bed, but

\footnotetext{
${ }^{*}$ Corresponding author

Email address: jtvanlew@fusion.ucla.edu (Jon T. Van Lew)
}

Preprint submitted to Fusion Engineering and Design current models lack predictive capability with full consideration for granular crushing, resettling, and interstitial gas.

The discrete element method (DEM) has emerged as an extremely powerful approach for interrogating and modeling evolving, transient, pebble-scale information in packed beds. DEM models have also been successfully coupled to volume-averaged computational fluid dynamics (CFD) models; coupled CFD-DEM models are capable of predicting temperature distributions in ceramic pebble beds with the slow-flowing interstitial purge gas while continuing to provide useful information on pebble-scale interactions. In this paper, we employ coupled CFD-DEM models with fragmentation modeling schemes to study the combined effects of initial packing fraction, crushed pebbles, gravity-influenced bed resettling after crushing events, and orientation of containing structures on temperature distributions in packed beds.

February 2, 2016 


\section{Numerical methodology}

With our DEM model, each pebble is treated as a perfectly elastic, smooth, non-cohesive sphere with mechanical interactions described by Hertzian contact laws where a conductance of heat is permitted through the contact area (see Ref. [1] and the references therein). Pebble interaction with fluid flow is captured via: a fluid drag coefficient, as calculated from the local fluid velocity, applied over pebble surfaces; and a local Nusselt number, calculated from passing fluid temperatures and velocities. The numerical technique is described in more detail in a previous study [2]. The position and temperature of every particle evolve with the explicit velocityVerlet time marching scheme.

The fluid-phase flow and temperature fields are calculated from transient, volume-averaged formulations of Navier-Stokes and energy equations calculated in computational cells that are overlaid on the DEM domain. Two-way coupling with the DEM model is achieved with a drag force source term in Navier-Stokes and an energy source term in the energy equation of the gas phase which are volumeaveraged summations of the drag and heat transfer coefficients calculated with DEM for all pebbles. We employ modeling tools of our previous study in which more details can be found [2].

\subsection{Simulation domain, boundary conditions, and material properties}

Two ITER-relevant volumes are considered in this study, sketched in Figures 1a and 1b. They are differentiated from each other by gravity's direction in the configuration. Because of their similarity, we use generic coordinate systems $(\chi, \zeta)$. Thus $\chi$-configurations, shown in Figure 1a, have $\chi=y$ and $\zeta=x$ while $\zeta$-configurations, Figure $1 \mathrm{~b}$, have $\chi=x$ and $\zeta=y$. The $\zeta$-configuration in this study is meant to represent the orientation of European Union's TBM [3], while the $\chi$ configuration is an orientation adopted by many other current TBM designs in ITER [4, 5].

In terms of the generic coordinates, outflow of bed heat to coolant is along $\zeta$. Constant temperature boundaries, $T_{w}$, exist at the edges of that dimension. As sketched in Figure 1, gravity resettling in the $\chi$ configuration will not allow gap formation between bed and wall. However, in the $\zeta$-config it is possible for a gap to form between top coolant walls and pebbles after gravity resettling. A constant nuclear heat rate was applied to every particle in the

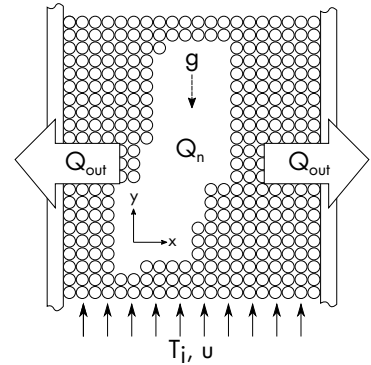

(a) $\chi$-configuration: heat removed in the $x$-direction.

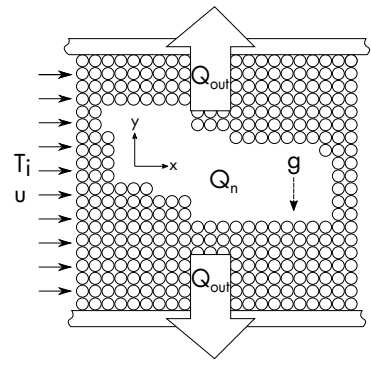

(b) $\zeta$-configuration: heat removed in the $y$-direction
Figure 1: Sketches of the two breeder orientations show that gravity settling will not allow gaps between pebbles and walls in the $\chi$-configuration. However for the $\zeta$-configuration, gravity-induced resettling can create a gap between pebbles and upper wall.

bed which is representative of the highest source term anticipated in current ITER designs of solid breeder blankets, $q^{\prime \prime \prime}=8 \times 10^{6} \mathrm{~W} \mathrm{~m}^{-3}$.

The simulation consists of pebbles of diameter $d_{p}=1 \mathrm{~mm}$, in beds filled to two initial packing fractions, $\phi_{1,2}=62,64 \%$. Mechanical properties of the pebbles are given in Table 1. To note is the Young's modulus chosen for pebbles in this study. In a past experimental study, Van Lew et al. found that individual pebbles behaved in a manner indicative of having a Young's modulus from 20 to $60 \%$ of values reported in literature for sintered blocks of $\mathrm{Li}_{2} \mathrm{TiO}_{3}$ and $\mathrm{Li}_{4} \mathrm{SiO}_{4}[6]$. Therefore to maintain some generality to this study, we have chosen a Young's modulus at a nominal value of $E=60 \mathrm{GPa}$ to generically represent either ceramic pebble.

Pebble bed widths in $\zeta$ are $20 \mathrm{~mm}$, a size comparable to breeding volumes of many ITER TBM designs [3-5]. Bed depths (in the $z$-direction, in/out of the page in Figures 1a and $1 \mathrm{~b}$ ) are $5 \mathrm{~mm}$, with periodic boundary conditions. Bed lengths in $\chi$ vary to accommodate 6000 pebbles, initially, with given initial packing fractions; the length is approximately $50 \mathrm{~mm}$. Virtual walls are placed at extents of $\chi$ and $\zeta$ dimensions. Walls in $\zeta$ had constant temperature boundaries of $T_{w, s}$, all walls have mechanical and thermal properties of structural steel, given in Table 2 .

Fluid domains, overlaid on DEM pebbles, have fluid inlet and outlet regions of lengths $10 \mathrm{~mm}$ and approximately $51 \mathrm{~mm}$, respectively. The side walls of the fluid domain were adiabatic in inlet and outlet regions and had constant tempera- 
ture boundaries where they contacted the pebble bed, $T_{w, f}$. Fluid entered with a constant velocity magnitude of $5 \mathrm{~cm} \mathrm{~s}^{-1}$ and constant temperature $T_{i}$. At present, temperature-dependencies of helium properties have not been incorporated into the model. Over the range of $400{ }^{\circ} \mathrm{C}$ to $900{ }^{\circ} \mathrm{C}$, increases in helium momentum and thermal diffusivities are both essentially linear and thus an arithmetic mean for properties over that range is used as a first approximation. Future models will incorporate temperature-dependence of fluid properties. Fluid transport properties are given in Table 3 .

Table 1: Mechanical and thermal properties of ceramic pebbles in the DEM domain. Aside from $E_{s}$, properties come from [7] with $\epsilon=0.2$

\begin{tabular}{lcc}
\hline Property & Symbol & Value \\
\hline Young's modulus $(\mathrm{GPa})$ & $E_{s}$ & 60.0 \\
Poisson ratio & $\nu_{s}$ & 0.24 \\
thermal conductivity $\left(\mathrm{W} \mathrm{m}^{-1} \mathrm{~K}^{-1}\right)$ & $k_{s}$ & 1.79 \\
diameter $(\mathrm{m})$ & $d_{p}$ & 0.001 \\
pebble-pebble friction coefficient & $\mu_{s}$ & 0.2 \\
pebble-wall friction coefficient & $\mu_{w}$ & 0.2 \\
heat capacity $\left(\mathrm{J} \mathrm{kg}^{-1} \mathrm{~K}^{-1}\right)$ & $c_{s}$ & $1.45 \times 10^{3}$ \\
thermal expansion coefficient $\left(\mathrm{K}^{-1}\right)$ & $\beta_{s}$ & $1.77 \times 10^{-5}$ \\
density $\left(\mathrm{kg} \mathrm{m}^{-3}\right)$ & $\rho_{s}$ & $3.44 \times 10^{3}$ \\
\hline
\end{tabular}

Table 2: Mechanical and thermal properties and boundary conditions of structural container in the DEM domain.

\begin{tabular}{lcc}
\hline Property & Symbol & Value \\
\hline Young's modulus $(\mathrm{GPa})$ & $E_{w}$ & 175.0 \\
Poisson ratio & $\nu_{w}$ & 0.30 \\
thermal conductivity $\left(\mathrm{W} \mathrm{m}^{-1} \mathrm{~K}^{-1}\right)$ & $k_{w}$ & 29.0 \\
wall temperature $(\mathrm{K})$ & $T_{w, s}$ & 573 \\
\hline
\end{tabular}

Table 3: Transport properties of helium and boundary conditions in the CFD domain; mean values over the temperature range $400^{\circ} \mathrm{C}$ to $900^{\circ} \mathrm{C}$.

\begin{tabular}{lcl}
\hline Property & Symbol & Value \\
\hline thermal conductivity $\left(\mathrm{W} \mathrm{m}^{-1} \mathrm{~K}^{-1}\right)$ & $k_{f}$ & $3.40 \times 10^{-1}$ \\
heat capacity $\left(\mathrm{J} \mathrm{kg}^{-1} \mathrm{~K}^{-1}\right)$ & $c_{f}$ & $5.19 \times 10^{3}$ \\
density $\left(\mathrm{kg} \mathrm{m}^{-3}\right)$ & $\rho_{f}$ & $5.38 \times 10^{-2}$ \\
kinematic viscosity $\left(\mathrm{m} \mathrm{s}^{-2}\right)$ & $\lambda_{f}$ & $8.52 \times 10^{-4}$ \\
thermal diffusivity $\left(\mathrm{m} \mathrm{s}^{-2}\right)$ & $\alpha_{f}$ & $1.28 \times 10^{-3}$ \\
wall temperature $(\mathrm{K})$ & $T_{w, f}$ & 573 \\
inlet temperature $(\mathrm{K})$ & $T_{i}$ & 573 \\
\hline
\end{tabular}

\subsection{Modeling crush events}

Models have been proposed in the past which translate experimental measurements of granular crushing into contact forces in an ensemble to predict granular crushing, e.g. Refs. [6, 8-11], but no validation has set any model apart as yet. Here, packed beds experience artificial pebble-crushing events for which a chosen percentage, $\eta$, of initial pebbles (at randomized locations) fragment. Four pebble crush percentages are used: $\eta=0,1,3,5 \%$. Numerical models of crush events themselves have received attention. Annabattula, Zhao, and Gan attempted to model a crushing event as either a reduction in radius of the crushed pebble or a reduction in Young's modulus [12-14]. Van Lew et al made similar simplifications when they considered a crushed pebble as being removed from the force network, thus being removed from the DEM domain [1].

In this work, we replace a parent pebble of radius $r$ with $N_{c}$ smaller daughter fragments, each of equal radius, $r_{c}$. After a crushing event, fragments are free to resettle through interstitial gaps in the pebble bed and original pebbles respond in kind with re-arrangement into a new metastable packing structure. To conserve volume between preand post-crush, we can relate the number of daughter fragments to the radius ratio between fragments and parent, $r^{*}=r_{c} / r$, as $N_{c}=\left(1 / r^{*}\right)^{3}$. Conservation of energy of crush event is enforced by setting the temperature of daughter fragments to the parent pebble at the moment of crushing.

Experimental studies of crushing brittle pebbles show many different modes of fragmentation, often with highly irregular sizes (see e.g. Ref.[15]). As a first effort, we compare the effect of fragmentation size by studying two different values, $r_{1}^{*}=0.352$ and $r_{2}^{*}=0.2$ which results in $N_{c, 1}=23$ and $N_{c, 2}=$ 125 daughters per crushed parent (at $\eta=5 \%$, for $r_{2}^{*}$ the system expands to 43200 pebbles).

As a consequence of volume conservation in a fragmentation event, the daughter fragments naturally cannot fit into the parent pebble without overlap. Numerically, in our fragmentation procedure, overlap and the associated high contact forces between the daughter fragments is permitted. Directly following a fragmentation event, a cut-off distance is applied to the velocity-Verlet integration of daughter fragments which prevents instabilities in position; e.g. in any given timestep fragments are only allowed to travel $x_{\text {cutoff }}$, regardless of the distance calculated in integration. A short time is permitted for the daughter fragments to relax away from the highly-overlapped state; relaxation time was a function of number of pebble fragments and 
pebble fragment size. Contact forces in the system were monitored and when average values returned to pre-fragmentation levels, the relaxation procedure concluded, cut-off distance was removed, and standard velocity-Verlet integration of daughter pebbles was reinstated.

\section{Numerical solvers}

Pebble contact and heat transfer models are packaged into LIGGGHTS, a modification of LAMMPS with powerful additions for granular material [16, 17]. The helium fluid domain is modeled with a modified PISO solver in the framework of OpenFOAM ${ }^{\circledR}$ from OpenCFD, Ltd 18. Coupling between LIGGGHTS and OpenFOAM is accomplished with open-source, modular $\mathrm{C}++$ libraries maintained by CFDEM Project [19].

\section{Results \& discussion}

In Figure 2, a bed of $64 \%$ initial packing with $5 \%$ of pebbles broken into fragments of size $r_{2}^{*}=0.2$ is shown; vectors of flow field, colored by fluid temperature, are seen moving through the bed. The inset image qualitatively demonstrates how the fluid field aids in equilibrating temperatures of fragments with neighboring larger pebbles in spite of light physical contact between pebbles.

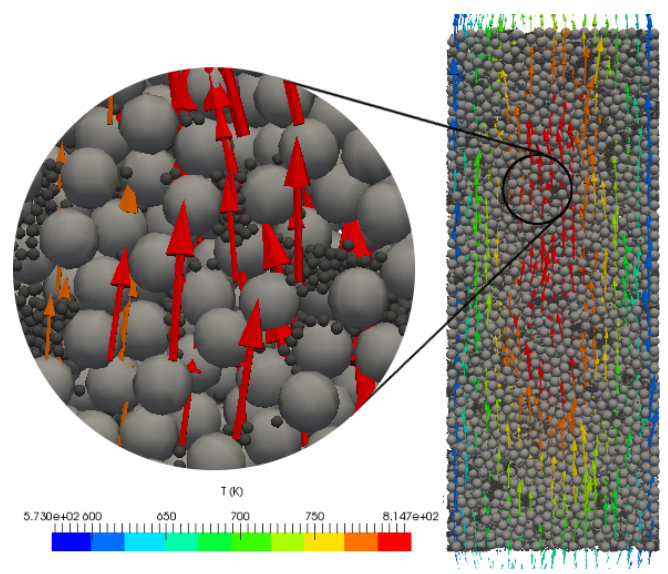

Figure 2: Showing the case for $\phi_{2}=0.64, \eta=5 \%$ with fluid velocity vectors colored by temperature. Inset image reveals size discrepancies between fragments and pebbles and the ensemble interaction with fluid flow.

Several cross-sections are created at mid-planes in $z$. Representative results are taken from $64 \%$ packings of $\zeta$ - and $\chi$-configurations and compared against their respective $\eta=5 \%, r_{2}^{*}$ conditions, shown in Figures 3 and 4 . The numbered zones of the beds will be discussed shortly.

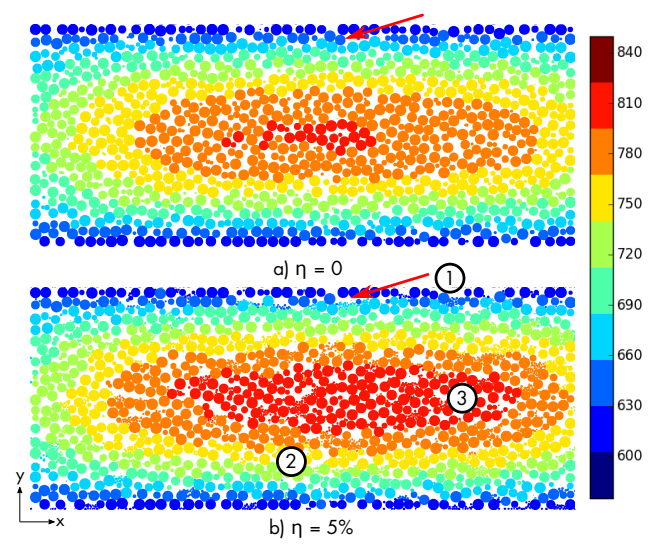

Figure 3: Cuts at the mid-plane of $z$ for $\zeta$-config beds. (a) bed initially packed to $\phi_{2}$, and (b) bed with crushing of $\eta_{5}$ pebbles with particle size $r_{2}^{*}$. Three important zones have been identified

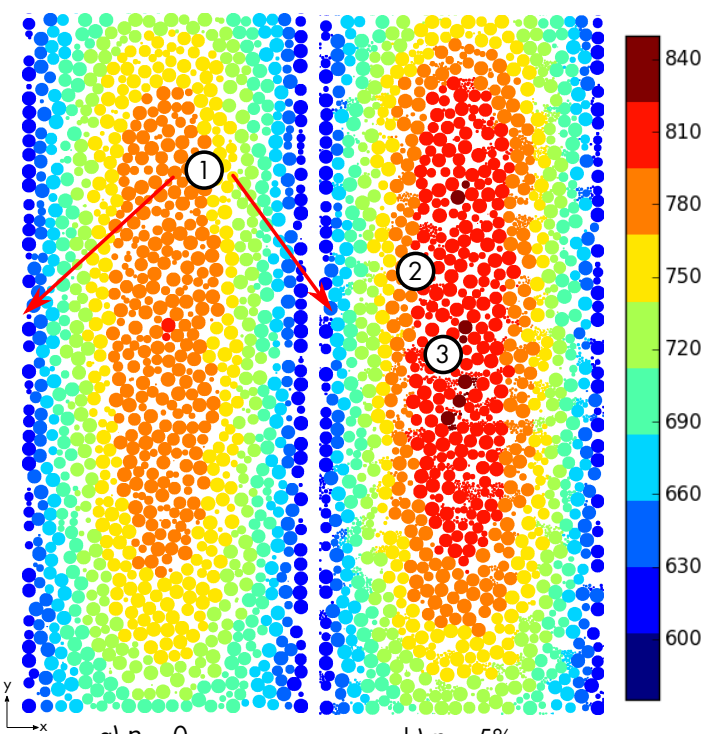

Figure 4: Cuts at the mid-plane of $z$ for $\chi$-config beds. (a) bed initially packed to $\phi_{2}$, and (b) bed with crushing of $\eta_{5}$ pebbles with particle size $r_{2}^{*}$. Three important zones have been identified

Wall offset-temperatures $\left(T-T_{w}\right)$ in beds are found in slices of width $\Delta \zeta$ along $\zeta$. Pebbleweighted mean values are found as $\langle T\rangle=$ $\frac{1}{V_{n}} \sum_{j}^{n}\left(T_{j}-T_{w}\right) V_{j}$, for $n$ pebbles of temperature $T_{j}$ consuming a total volume $V_{n}$ in the slice. Demonstrative cases of $\phi_{2}=0.64$ with $r_{2}^{*}=0.2$ are given 
in Figure 5 as functions of granular crushing percentage.

Following the formulations described by Gan \& Kamlah [20], and Gan et al.[21], we report the overall hydrostatic pressure of pebble beds, $p=\sigma_{i i} / 3$, at steady-state heating. The values are normalized against initial $(\eta=0)$ beds for respective configurations. Hydrostatic pressure is given in Figure 6 .

A total mean bed temperature is found from all pebbles in the system as $\langle T\rangle_{t o t}=\frac{1}{V_{N}} \sum_{j}^{N}\left(T_{j}-\right.$ $\left.T_{w}\right) V_{j}$ where $N$ is the total number of ensemble pebbles. The total mean bed temperature is also normalized against initial packing cases of each respective set of beds. Results for all beds are given in Figure 7. Maximum temperature rises of every bed are also found, $T_{m}=\max (T)-T_{w}$, and normalized against the maximum temperature in the initial packing of each respective set of beds. To avoid aberrant results from a bed which might have a single, very high temperature pebble, the maximum temperature, $\max (T)$, is calculated as a mean value of the 50 highest temperature pebbles. The results are given in Figure 8. In addition to total mean bed temperature, maximum temperature rise is also an important factor in evaluation of a pebble bed.

Lastly, we consider how far pebble fragments travel in the bed after a crushing event. Total displacements from the moment of crush event to final resting, $|\Delta h|$, are normalized against the original pebble diameter, $|\Delta h| / d_{p}$. Histograms for $64 \%$ packing fractions of $\chi$ - and $\zeta$-configurations at $5 \%$ pebble crushing with fragment sizes $r_{2}^{*}$ are given in Figure 9. These two beds saw the most settling, all other beds tested saw considerably less fragment travel.

Analyzing results of all the pebble beds in this study revealed two main contributors to bed temperatures with resettling from pebble crushing: final settling location of fragment particles and overall contact force relaxation. The two interacting contributors were found to be expressed to different extents depending on crush amount and bed configuration.

Hydrostatic pressure (a global measure of interparticle contact forces) is predominately a function of initial packing fraction alone, as seen in Figure 6 . Smaller initial packing fractions had their internal hydrostatic pressure reduced more rapidly as pebbles crushed in the ensemble. Breeder orientation appears to have less impact on stress relief than size of crush fragments. Due to the inter-connected

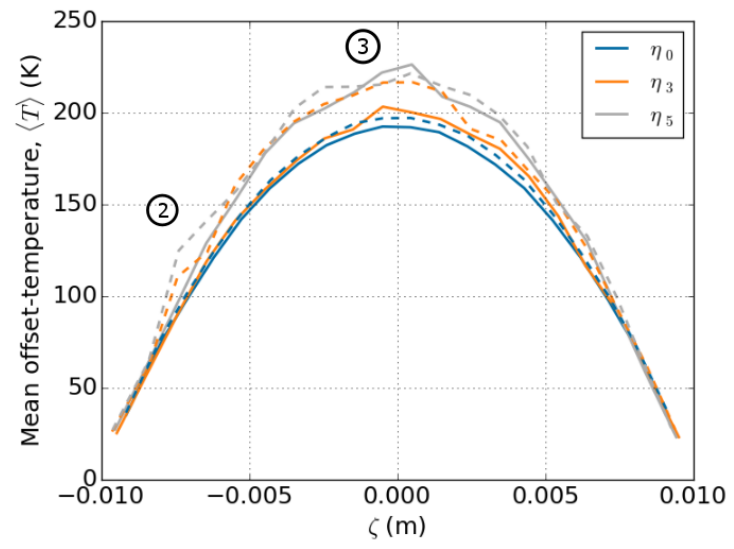

Figure 5: Mean offset-temperature profiles, $\langle T\rangle$, along $\zeta$ in beds with initial packing fractions $\phi_{2}=0.64$, fragmentation sizes were $r_{2}^{*}$. Solid lines are $\chi$ configurations, dashed lines are $\zeta$ configurations. Fragmentation settling of $\zeta$-configs are seen in 'lumps' near Zone (2) and in the $\chi$-config spike in zone (3).

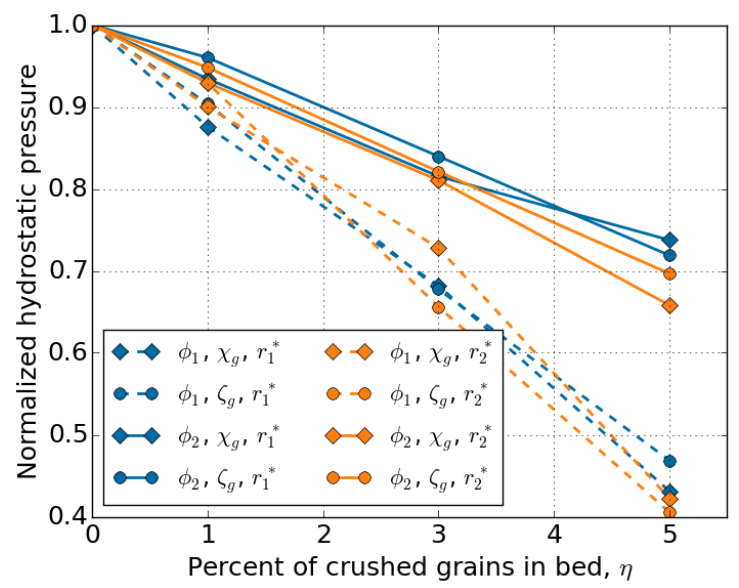

Figure 6: Dashed lines represent the lower packing fraction, $\phi_{1}=0.62$, solid lines are $\phi_{2}=0.64$. Markers are: $\circ$ for $\zeta$-config, $\diamond$ for $\chi$-config. Color differentiates the fragment radius ratio. Contact force relaxation is more rapid for lower packing fractions.

nature of the force network, and geometry of beds studied here, resettling in beds and contact force relaxation is uniform throughout the beds. Thus we expect reductions in contact forces to directly result in an overall increase of bed temperatures. This is reflected in the curves of Figure 7 . Total mean temperatures of $\phi_{1}=0.62$ beds increased between 16 to $19 \%$ at $\eta=5 \%$. Yet beds initially packed to $\phi_{2}=0.64$ increased by only 10 to $13 \%$ at the same value of crushed pebble amount.

Fragment settling location, on the contrary, is 


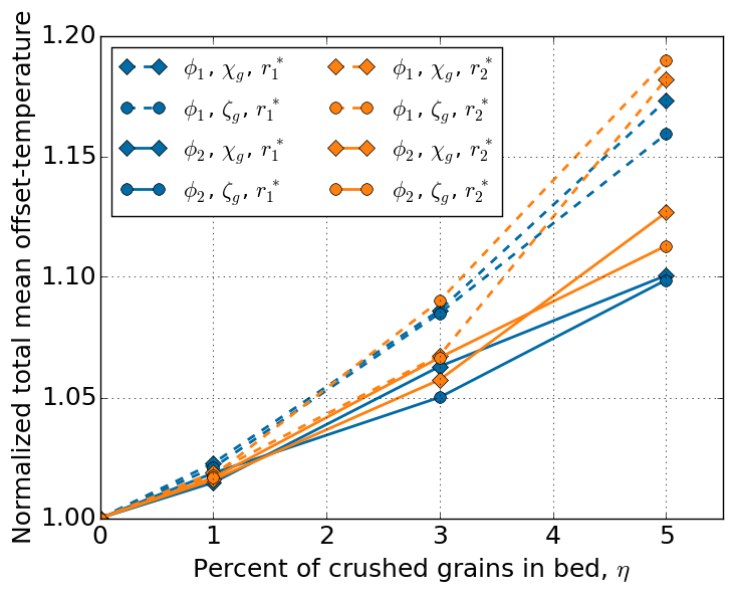

Figure 7: Dashed lines represent the lower packing fraction, $\phi_{1}=0.62$, solid lines are $\phi_{2}=0.64$. Markers are: $\circ$ for $\zeta$-config, $\diamond$ for $\chi$-config. Color differentiates the fragment radius ratio. Lower packing fraction is the most dominant parameter for overall bed temperature. Amongst the same packing fraction, fragment size is most influential factor.

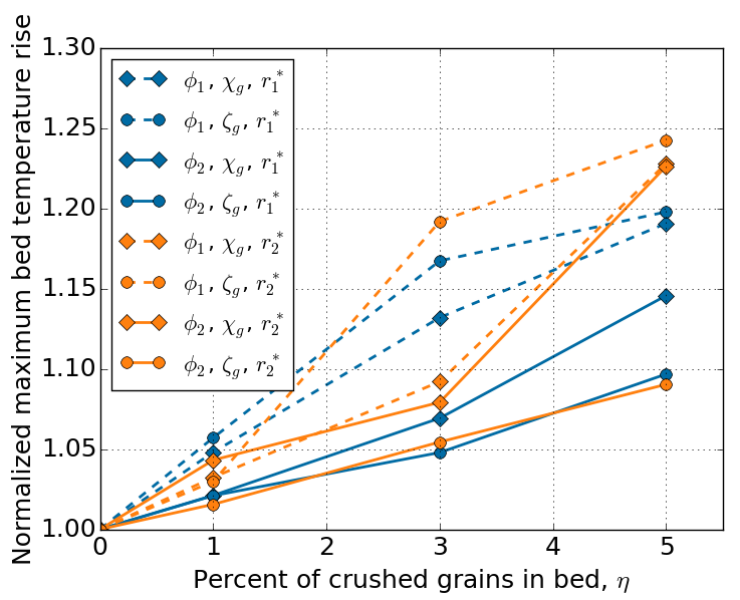

Figure 8: Dashed lines represent the lower packing fraction, $\phi_{1}=0.62$, solid lines are $\phi_{2}=0.64$. Markers are: $\circ$ for $\zeta$-config, $\diamond$ for $\chi$-config. Color differentiates the fragment radius ratio. The dominant parameter influencing maximum bed temperature varies as a function of the number of crushed pebbles in the bed.

strongly dependent on fragment size and breeder orientation. Larger pebble fragments generally did not travel far, settling loosely in regions near the point of fragmentation. Smaller fragments were seen to be capable of traveling much further through interstitial gaps between pebbles before also coming to rest with loose settlings. The looseness of the fragment settling is indicated by their ensemble-average coordination number (counting

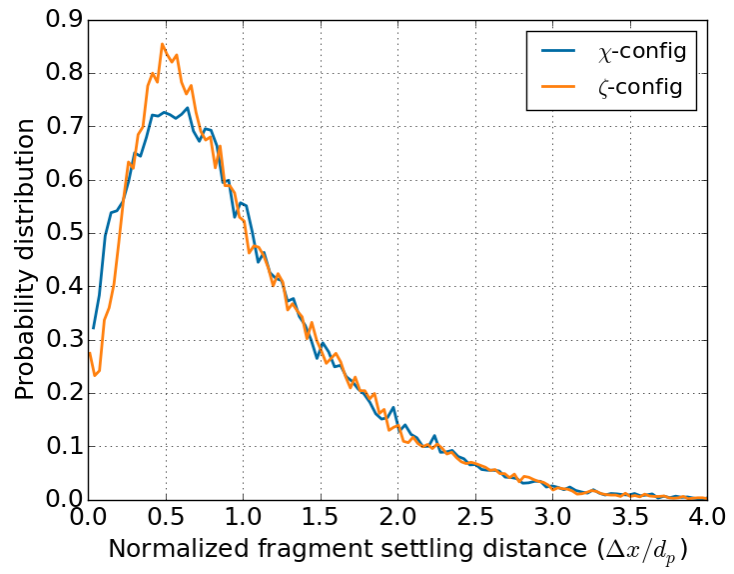

Figure 9: Normalized displacement histograms for fragment sizes of $r_{2}^{*}=0.2$ with $\eta=5 \%$. $\chi$-config: $59.7 \%$ of fragments travel up to $1 \mathrm{~mm}$ and $8.2 \%$ travel more than $2 \mathrm{~mm}$; $\zeta$-config: $60.7 \%$ of fragments travel up to $1 \mathrm{~mm}, 7.9 \%$ travel more than $2 \mathrm{~mm}$.

only the fragments) for $r_{1} *$ and $r_{2}^{*}$, respectively, as $\langle Z\rangle=3.2$ and $\langle Z\rangle=2.8$. Contacts with walls or floors are not counted in this coordination number. A consequence of loose packings of fragments is poor thermal conductance to neighboring pebbles. Fragment temperatures are therefore mostly regulated by convection with interstitial helium and their influence on bed temperatures is much more complex.

To identify the effects of fragment settling, we look to Figure 9 and the several zones demarcated in the results of Figures 3 and 4 . The majority of fragments, even in this case of smallest fragment size and largest crushing amount, remain approximately at the location of the parent pebble; for both configurations, approximately $60 \%$ travel less than 1 pebble diameter $(1 \mathrm{~mm})$. However, in both configurations, approximately $8 \%$ of fragments travel more than 2 diameters, and those pebbles have a significant impact on the ensembles overall thermal response.

In $\zeta$-configuration beds, pebbles traveling more than a few pebble diameters will move between colored isotherms drawn in Figure 3. For example we can see the fragment group identified in Zone (1) as moving downward, away from the top wall. Similarly, when pebbles in Zone (3) are crushed, some of the fragments tumble downward into Zone (2) before coming to rest where they continue to receive volumetric heating. Thus the fragments, with poor thermal conductance, increase heating in the 
regions where they settle. The effect is seen in the $\eta=3,5 \%$ temperature profiles in Figure 5 that are asymmetric with lower temperatures in the top half (above Zone (3)) and higher temperatures in the region near Zone (2).

In contrast, $\chi$-config beds respond much differently to pebble fragment settling. We again see from cross-sections in Figure 4 a pebble identified in Zone (1) that breaks but remains in that zone after settling. The trend continues in other regions of the bed. When pebbles are crushed in the $\chi$-config beds, gravity causes them to fall downward but remain generally in the same isotherm, as drawn in Figure 4. According to Figure 5, the effect of pebble crushing has little effect up to $3 \%$ of damaged pebbles. But suddenly at $\eta=5 \%$, the combination of reduced overall bed pressure and fragmentation heating causes the maximum bed temperature to jump above all other $\phi=64 \%$ beds (see Figure 5 and Figure 8). The temperature increase in this $\chi$-config bed is due to the accumulation of pebble fragments that remain in Zone (3), only tumbling to lower heights. Helium that enters the $\chi$-configuration bed reaches higher temperatures more quickly due to the increased heating from fragments which settled at lower heights of $y$. Helium then continues to heat from the many fragments in Zone (3) which ultimately results in the highest maximum bed temperatures (for the given packing fraction). This can also be seen with comparison between Figures 3 and 4: the $\chi$-config bed reaches the $780 \mathrm{~K}$ contour at a much lower height than the $\zeta$-configuration.

\section{Conclusions}

We conducted several multi-scale simulations of granular heat transfer using coupled CFD-DEM simulations of representative tritium-breeding ceramic pebble bed volumes with parametric variations of: bed orientation with respect to gravity, pebble crushing amount, initial packing fraction, and crushed fragmentation size.

There was one general trend observed that reiterates past conclusions from solid breeder research. Namely, more persistent behavior is witnessed in pebble beds with higher initial packing fractions. In this study, the most dominant parameter observed to affect temperatures in pebble beds was the initial packing fraction: beds with higher initial packing fraction had smaller increases in bed temperatures due to pebble crushing. We therefore conclude that manual densification, from either long-term vibration packing or load-induced pre-compaction, must be done to ceramic pebble bed volumes to gain some temperature control during operation in a fusion reactor. To achieve packing fractions of $64 \%$ in the relatively small sizes of this study, for example, a load of over $1 \mathrm{MPa}$ was necessary. In the assembly of tritium breeding modules, it must be kept in mind that similar pre-compaction may need to be performed.

As stated earlier, a concern with the $\zeta$ design is the possibility of gap formation between pebble bed and upper walls after bed resettling, particularly after pebble fragmentation. In this study, we found pebble beds initially packed to $\phi=62 \%$ experienced the highest increases in both total average bed temperature as well as maximum temperature rise. The comparably looser packing allowed a quick reduction in hydrostatic pressures and consequently a reduction in heat conduction to the upper wall. Nevertheless, no gaps were detected even at $5 \%$ of pebbles crushed. However, temperatures in pebble beds packed to $64 \%$ showed a resistance to fragmentation; overall average temperatures were comparable to $\chi$-configurations, and in fact these EU-style beds had the lowest maximum temperatures for beds with many crushed pebbles. We showed that freedom of fragments to travel between zones in these beds prevented a build-up of loose fragments (and thereby avoided build-up of heating) in the hottest regions.

As for the $\chi$-configurations, we found that when there were not many broken pebbles $(\eta \leq 3 \%)$, these beds generally had lower temperatures in comparison to similar $\zeta$-config beds. But as $\eta$ went above $3 \%$ for many of the beds, the averaged bed temperature and, importantly, the maximum temperature rise actually jumped above the $\zeta$-configurations. We showed that for these beds it was the inability for fragments to move between zones which left many small fragments to settle in the hottest region, further contributing to heating.

From the results we have shown, it is obvious that pebble crushing and bed resettling effects on temperature are complicated, non-linear responses and are particular to breeder design and ceramic material employed. We found indications of certain operational spaces for which different designs responded less severely to pebble crushing. For instance, from the sole point of view of temperature response in pebble beds, if one were to employ a material known to have a limited crush strength, one might accept 
that many pebbles could break (at least up to $5 \%$, as studied here) over the life of the breeder and choose to employ the $\zeta$-style which avoided large increases in temperature after long operation of the breeder. Alternatively, if one had a ceramic material with a larger crush strength, the $\chi$-design would be preferable as it generally retained lower overall and maximum bed temperatures when fewer pebbles in the ensemble were crushed.

It must be pointed out that the findings discussed here are concerned primarily with temperature distribution, without consideration for other consequences of pebble crushing such as blocking of helium purge, and thereby tritium extraction, by clogging from fragment dust or particulates. Clogging of purge flow is specific to each pebble bed design and must receive future attention in its own right.

This study was performed on some generic geometries and has provided some generalized conclusions. But in light of the pebble beds' complex responses, as breeder designs continue to evolve into their final form before deployment in ITER, CFDDEM models should continuously be employed to study the specific thermomechanical responses to pebble crushing and bed resettling unique to each design.

\section{Acknowledgments}

This work was performed with support from the US Department of Energy, Office of Fusion Energy Sciences, under Grant No. DE-FG02-86ER52123.

\section{References}

[1] J. T. Van Lew, A. Ying, M. A. Abdou, A discrete element method study on the evolution of thermomechanics of a pebble bed experiencing pebble failure, Fusion Engineering and Design 89 (2014) 1151-1157.

[2] J. T. Van Lew, A. Ying, M. Abdou, Coupling Discrete Element Models of Ceramic Breeder Pebble Beds to Thermofluid Models of Helium Purge Gas Using Volume-Averaged Navier-Stokes and the LatticeBoltzmann Method, Fusion Science and Technology 68 (2015).

[3] F. Hernández, M. Kolb, M. Ilić, a. Kunze, J. Németh, a. Von Der Weth, Set-up of a pre-test mock-up experiment in preparation for the HCPB Breeder Unit mock-up experimental campaign, Fusion Engineering and Design 88 (2013) 2378-2383.

[4] S. Cho, M.-Y. Ahn, D. H. Kim, E. S. Lee, S. Yun, N. Z. Cho, K. J. Jung, Current status of design and analysis of Korean Helium-Cooled Solid Breeder Test Blanket Module, Fusion Engineering and Design 83 (2008) $1163-1168$.
[5] K. M. Feng, C. H. Pan, G. S. Zhang, T. Y. Luo, Z. Zhao, Y. J. Chen, Y. J. Feng, X. F. Ye, G. Hu, K. H. He, R. W. Niu, Z. X. Li, P. H. Wang, B. Xiang, L. Zhang, Q. J. Wang, F. C. Zhao, Q. X. Cao, F. Wang, T. Yuan, G. Y. Zheng, Y. Liu, Y. Zhong, M. C. Zhang, Progress on design and R\&D for helium-cooled ceramic breeder TBM in China, Fusion Engineering and Design 87 (2012) $1138-1145$.

[6] J. T. Van Lew, Y.-H. Park, A. Ying, M. Abdou, Modifying Young's modulus in DEM simulations based on distributions of experimental measurements, Fusion Engineering and Design (2015).

[7] P. J. Gierszewski, Review of properties of lithium metatitanate, Fusion Engineering and Design 39-40 (1998) 739-743.

[8] Y. Gan, M. Kamlah, H. Riesch-Oppermann, R. Rolli, P. Liu, Crush probability analysis of ceramic breeder pebble beds under mechanical stresses, Journal of $\mathrm{Nu}-$ clear Materials (2010) 10-13.

[9] A. R. Russell, D. Muir Wood, M. Kikumoto, Crushing of particles in idealised granular assemblies, Journal of the Mechanics and Physics of Solids 57 (2009) 12931313.

[10] S. Zhao, Y. Gan, M. Kamlah, Spherical ceramic pebbles subjected to multiple non-concentrated surface loads, International Journal of Solids and Structures 49 (2012) 658-671.

[11] R. K. Annabattula, Size-Dependent Crush Analysis of Lithium Orthosilicate Pebbles, Fusion Science \& Technology 66 (2014).

[12] R. K. Annabattula, Y. Gan, M. Kamlah, Mechanics of binary and polydisperse spherical pebble assembly, Fusion Engineering and Design (2012) 2-7.

[13] S. Zhao, Y. Gan, M. Kamlah, Failure initiation and propagation of $\mathrm{Li} 4 \mathrm{SiO} 4$ pebbles in fusion blankets, $\mathrm{Fu}-$ sion Engineering and Design 88 (2013) 8-16.

[14] R. K. Annabattula, Y. Gan, S. Zhao, M. Kamlah, Mechanics of a crushable pebble assembly using discrete element method, Journal of Nuclear Materials 430 (2012) 90-95.

[15] S. Wu, K. Chau, T. Yu, Crushing and fragmentation of brittle spheres under double impact test, Powder Technology 143-144 (2004) 41-55.

[16] S. Plimpton, Fast Parallel Algorithms for Short-Range Molecular Dynamics, Journal of Computational Physics 117 (1995) 1-19.

[17] C. Kloss, C. Goniva, A. Hager, S. Amberger, S. Pirker, Models, algorithms and validation for opensource DEM and CFD-DEM, Progress in Computational Fluid Dynamics 12 (2012) 140-152.

[18] OpenCFD Ltd., OpenFOAM - The Open Source CFD Toolbox (2014).

[19] C. Goniva, C. Kloss, N. G. Deen, J. A. Kuipers, S. Pirker, Influence of Rolling Friction on Single Spout Fluidized Bed Simulation, Particuology 10 (2012) 582591.

[20] Y. Gan, M. Kamlah, Discrete element modelling of pebble beds: With application to uniaxial compression tests of ceramic breeder pebble beds, Journal of the Mechanics and Physics of Solids 58 (2010) 129-144.

[21] Y. Gan, F. Hernandez, D. Hanaor, R. K. Annabattula, M. Kamlah, P. Pereslavtsev, Thermal Discrete Element Analysis of EU Solid Breeder Blanket Subjected to Neutron Irradiation, Fusion Science \& Technology 66 (2014). 